\title{
Salud mental de los mexicanos durante la pandemia de COVID-19
}

\section{Cristina Rodríguez-Hernández, ${ }^{1 *}$ Omar Medrano-Espinosa ${ }^{2}$ y Ariadne Hernández-Sánchez ${ }^{3}$}

${ }^{1}$ Hospital Ángeles Mocel, Ciudad de México; ${ }^{2}$ Instituto de Seguridad y Servicios Sociales de los Trabajadores del Estado, Hospital Regional de Alta Especialidad Bicentenario de la Independencia, Estado de México; ${ }^{3}$ Universidad Nacional Autónoma de México, Facultad de Estudios Superiores Iztacala, Sistema de Universidad Abierta y a Distancia, Estado de México. México

\section{Resumen}

Introducción: La pandemia de COVID-19 también ha afectado la salud mental. Objetivo: Evaluar la salud mental de la población mexicana durante la pandemia de COVID-19 mediante la medición de síntomas de estrés, depresión, ansiedad, insomnio y resiliencia. Métodos: Estudio observacional, descriptivo y transversal. Con una encuesta se recabaron datos sociodemográficos y se aplicaron la Depression Anxiety and Stress Scale 21 (DASS 21), la Escala Atenas de Insomnio y la Escala de Resiliencia 14 Ítems (RS-14). Se obtuvieron medidas de tendencia central y de dispersión en las variables cuantitativas, así como frecuencias en las cualitativas. En el análisis bivariado se utilizó la prueba de $\chi^{2}$; el nivel alpha fue 0.05. Resultados: Se analizaron 1667 individuos con edad media de $33.78 \pm 10.79$ años. En la DASS 21 se encontró una media de 9.7 puntos (normal), 7.10 para ansiedad (normal) y 6.73 para depresión (normal). La Escala Atenas de Insomnio presentó una media de 9.33 puntos (alteración moderada) y la RS-14, 69.13 (resiliencia alta). Conclusiones: La intensidad de la sintomatología fue menor a la esperada en comparación con la registrada en otras poblaciones, probablemente por la alta resiliencia de la población mexicana.

PALABRAS CLAVE: COVID-19. Estrés. Depresión. Ansiedad. Insomnio. Resiliencia.

\section{Mental health of the Mexican population during the COVID-19 pandemic}

\begin{abstract}
Introduction: The COVID-19 pandemic has also affected mental health. Objective: To evaluate Mexican population mental health during the COVID-19 pandemic by measuring symptoms of stress, depression, anxiety and insomnia, as well as resilience. Methods: Cross-sectional, descriptive, observational study. A survey was carried out to collect sociodemographic data, and the Depression Anxiety and Stress Scale 21 (DASS 21), Athens Insomnia Scale and the 14-item Resilience Scale (RS-14) were applied. Central tendency and dispersion measures were obtained for quantitative variables and frequencies for qualitative variables. The chi-square test was used for bivariate analysis; alpha level was 0.05. Results: 1,667 individuals with a mean age of $33.78 \pm 10.79$ years were analyzed. On DASS 21, a mean of 9.7 points (normal) was found, as well as 7.10 for anxiety (normal) and 6.73 for depression (normal). In the Athens Insomnia Scale, a mean of 9.33 points (moderate alteration), and in the RS-14 scale, 69.13 points (high resilience) were obtained. Conclusions: Symptoms' intensity was lower than expected in comparison with that recorded in other populations, probably due to the high levels of resilience of the Mexican population.
\end{abstract}

KEY WORDS: COVID-19. Stress. Depression. Anxiety. Insomnia. Resilience.

Correspondencia:

*Cristina Rodríguez-Hernández

E-mail: crispasha@gmail.com

DOI: 10.24875/GMM.20000612
Gac Med Mex. 2021;157:228-233

Disponible en PubMed

www.gacetamedicademexico.com

CC BY-NC-ND (http://creativecommons.org/licenses/by-nc-nd/4.0/) 


\section{Introducción}

En marzo de 2020, la Organización Mundial de la Salud declaró a COVID-19 como una pandemia; en respuesta, los gobiernos de cada país dictaron medidas sanitarias conducentes a disminuir el número de contagios entre la población, como el aislamiento o distanciamiento social. ${ }^{1,2}$ Estas medidas fueron observadas como una variable con efectos psicológicos negativos de carga afectiva, comportamental y cognitiva. ${ }^{3,4}$

Además, la alerta sanitaria por COVID-19 desencadenó eventos estresantes tales como miedo al contagio, incertidumbre por la afectación de las finanzas personales, xenofobia, excesiva exposición a medios (infodemia), pánico a la muerte y temor al fallecimiento de seres cercanos, entre otros. ${ }^{5-7}$ De acuerdo con el informe COVID-19 y necesidades en salud mental, publicado por la Organización de las Naciones Unidas, se reconoció que de no abordar de forma pertinente y oportuna el impacto de esta pandemia sobre la salud mental de la población mundial, se derivará en una crisis de dimensiones incontrolables. ${ }^{8}$

Los estudios realizados a diferentes poblaciones alrededor del mundo han puesto en evidencia el impacto que la pandemia de COVID-19 tiene sobre la salud mental: por un lado, activa mecanismos adaptativos como la resiliencia y el afrontamiento positivo $y$, por el otro, pone en marcha mecanismos desadaptativos como trastornos de estrés, ansiedad, depresión, trastornos del sueño, de la conducta alimentaria, consumo excesivo de alcohol, tabaco y suicidio. 9-19

El objetivo de este estudio fue evaluar la salud mental de la población mexicana durante la contingencia por COVID-19 mediante la medición de síntomas de estrés, depresión, ansiedad, insomnio y resiliencia (medida por estrés percibido).

\section{Métodos}

Con el fin de conocer el impacto emocional que la pandemia de COVID 19 tiene en la población mexicana, durante mayo y junio de 2020 se aplicó una encuesta realizada en la aplicación Google Forms, la cual constaba de un cuestionario para recabar datos sociodemográficos y de tres escalas:

- Depression Anxiety and Stress Scale 21 (DASS 21), para medir la prevalencia de los síntomas de ansiedad, depresión e intensidad de estrés.
- Escala Atenas de Insomnio, para medir la prevalencia de dicho síntoma.

- Escala de Resiliencia 14 Ítems (RS-14).

Esta encuesta fue enviada de forma libre a través de diferentes plataformas electrónicas; al inicio de esta se solicitaba el consentimiento informado del usuario para utilizar la información con fines de investigación.

El análisis estadístico se realizó con medidas de tendencia central y de dispersión para variables cuantitativas y frecuencias para variables cualitativas. En el análisis bivariado se utilizó la prueba de $\chi^{2}$. El nivel alpha usado fue 0.05 . Se utilizó el programa estadístico SPSS versión 21.

\section{Resultados}

Se obtuvo una cohorte de 1667 pacientes con edad media de 33.78 años \pm 10.790 y rango de edad de 18 a 83 años; $81.6 \%$ de los participantes fue del sexo femenino y $18.4 \%$, del masculino. La escolaridad predominante fue la licenciatura (50.2\%), en ocupación prevaleció el desarrollo profesional (47.9\%) y los estados civiles predominantes fueron soltero $50 \%$ y casado $40.5 \%$.

Se encontró que, a lo largo de la vida, $58.8 \%$ de la población había recibido algún tipo de atención en el área de la salud mental, ya fuera por psicología o psiquiatría; las patologías más prevalentes fueron depresión (36.3\%) y ansiedad (37.7\%), como se detalla en la Tabla 1.

En relación con las escalas clinimétricas aplicadas, en la DASS 21 se encontró media de 9.7 para estrés, 7.10 para ansiedad y 6.73 para depresión, que califican en el rango de normalidad. Solo en la subescala de ansiedad se encontraron puntuaciones máximas, mientras que la máxima puntuación en depresión y estrés se encontró en el rango de afectación moderada. En la Escala Atenas de Insomnio, la media fue $9.33 \pm 5.051$ puntos, indicativa de alteración moderada del sueño. La escala RS-14 presentó una media de $69.13 \pm 19.184$ puntos, correspondiente a resiliencia alta (64 a 81 puntos) (Tabla 2).

Se utilizó $\chi^{2}$ para establecer asociación entre los antecedentes de sintomatología y la presencia actual de la misma; se encontró asociación estadísticamente significativa en las personas que tenían antecedentes de haber recibido atención por síntomas de depresión, ansiedad e insomnio y quienes durante mayo y junio presentaron sintomatología. La asociación entre 
Tabla 1. Prevalencia de trastornos psiquiátricos

\begin{tabular}{|l|c|c|}
\hline & n & $\%$ \\
\hline Depresión & 609 & 36.3 \\
\hline Ansiedad & 632 & 37.7 \\
\hline Trastorno obsesivo compulsivo & 70 & 4.2 \\
\hline Insomnio & 200 & 11.9 \\
\hline Trastorno por déficit de atención e hiperactividad & 39 & 2.3 \\
\hline Adicciones & 41 & 2.4 \\
\hline Trastorno de la personalidad & 69 & 4.1 \\
\hline Trastorno psicótico & 22 & 1.3 \\
\hline Deterioro cognitivo & 32 & 1.9 \\
\hline
\end{tabular}

Tabla 2. Escala DASS 21

\begin{tabular}{|l|c|c|c|}
\hline & Estrés & Ansiedad & Depresión \\
\hline Media & 9.70 & 7.10 & 6.73 \\
\hline Mediana & 9.00 & 6.00 & 5.00 \\
\hline Puntuación mínima & 1 & 1 & 1 \\
\hline Puntuación máxima & 21 & 21 & 18 \\
\hline
\end{tabular}

los niveles de estrés y la sintomatología de ansiedad no fue estadísticamente significativa (Figuras 1 a 3 ).

\section{Discusión}

A nuestro saber, los resultados presentados en este trabajo son los primeros obtenidos en relación con salud mental en población mexicana durante el periodo de contingencia por COVID-19.

Llama la atención que la población se percibe como altamente resiliente, esto quiere decir que tiene la capacidad de asumir con flexibilidad situaciones límite y sobreponerse a ellas, tal como lo demuestran los resultados obtenidos en RS-14 y que coinciden con lo mencionado por Palomar Gómez, quien señala alta resiliencia en los mexicanos. Autores como Fadah Sandman y González Valdez han encontrado que esta resiliencia es mayor en las mujeres que en los hombres, lo cual es congruente con lo encontrado en este estudio. ${ }^{20-22}$

Los síntomas percibidos de depresión, ansiedad y estrés contrastan con los reportados en otras poblaciones: Ustun menciona que la población turca presentó mayores índices de síntomas depresivos durante el confinamiento, lo cual fue más evidente en las mujeres; por su parte, las puntuaciones en ansiedad se ubicaron dentro de los parámetros normales, lo que difiere de lo reportado por Salari en población china, en la cual se reportó un incremento hasta de $30 \%$ en la incidencia de síntomas de ansiedad. ${ }^{23,24}$

Al realizar una comparación entre los pacientes con antecedente de algún trastorno de ansiedad o depresión y quienes nunca habían sido diagnosticados, se observó que la población sin antecedente de diagnóstico presentó puntuaciones más altas para la sintomatología. Lo anterior puede explicarse por tres situaciones:

- La primera y más relevante es la concordancia con las altas puntuaciones de resiliencia que presenta la población mexicana ante situaciones de estrés.

- En segundo lugar, el poco uso de servicios de salud mental ante la presencia de estas patologías. Es decir, se presume que estos pacientes presentaban sintomatología antes de la pandemia y no recibieron atención.

- Por último, que quienes reportaron un padecimiento previo podían estar bajo tratamiento, lo que impidió que la severidad de la sintomatología aumentará.

Finalmente, en relación con la Escala Atenas de Insomnio se demostró que la calidad y cantidad de sueño se ha visto gravemente afectada. ${ }^{25,26}$

\section{Conclusiones}

La pandemia de COVID-19 no solo está afectando la salud física de la población; la salud mental y el estado de bienestar de las personas también se alteran, lo que demanda atención prioritaria por parte de los especialistas en salud. Lo anterior implica un cambio en la práctica clínica que permita garantizar el cuidado y soporte de las personas con padecimientos mentales previos, así como de la población que comienza a padecer las consecuencias psicosociales de la pandemia de COVID-19. Ello representa un reto para la población y los sistemas de salud en términos de resiliencia. La generación de información basada en evidencia permitirá detectar la prevalencia de afectación en el dominio de la salud mental de la población mexicana, con el objetivo de generar estrategias de abordaje clínico.

Es importante mencionar que en este estudio se observó mayor afectación en las personas que no habían tenido previamente alguna alteración en la 


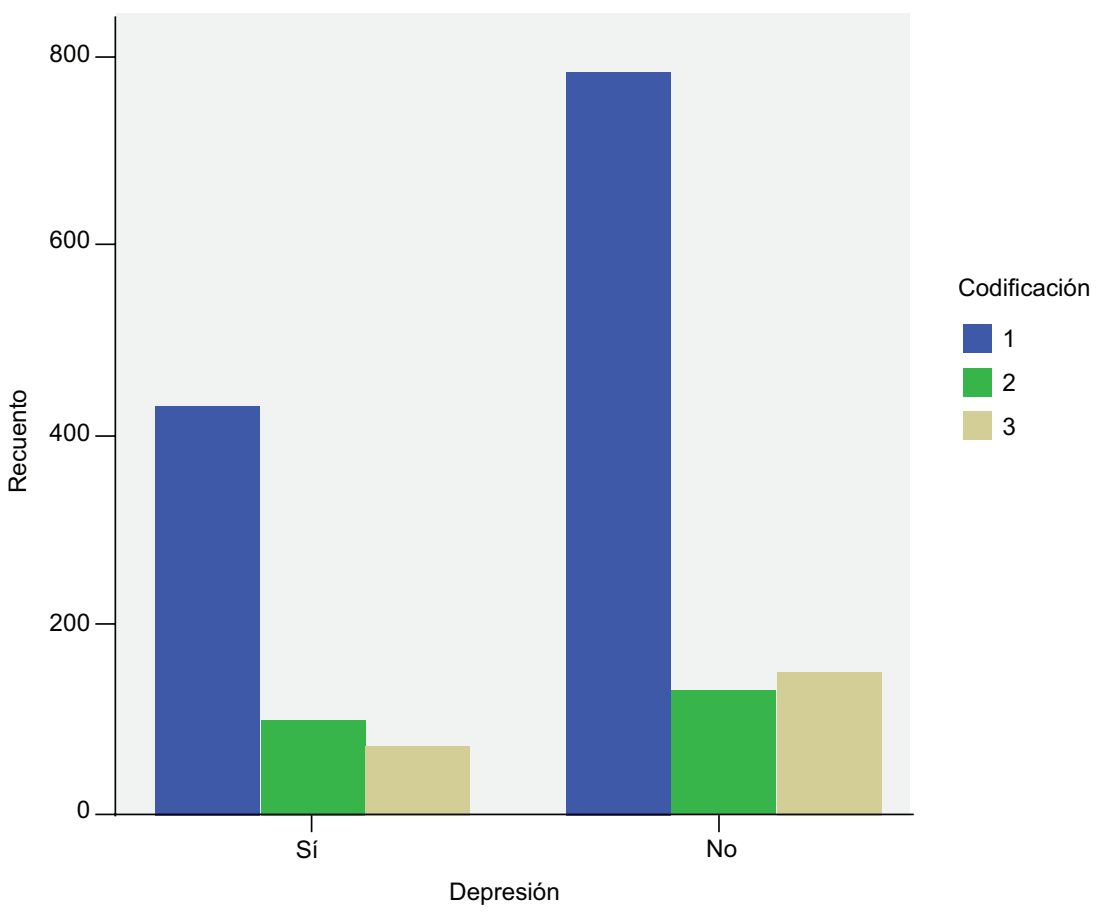

Figura 1. Asociación entre antecedentes de depresión y síntomas actuales.

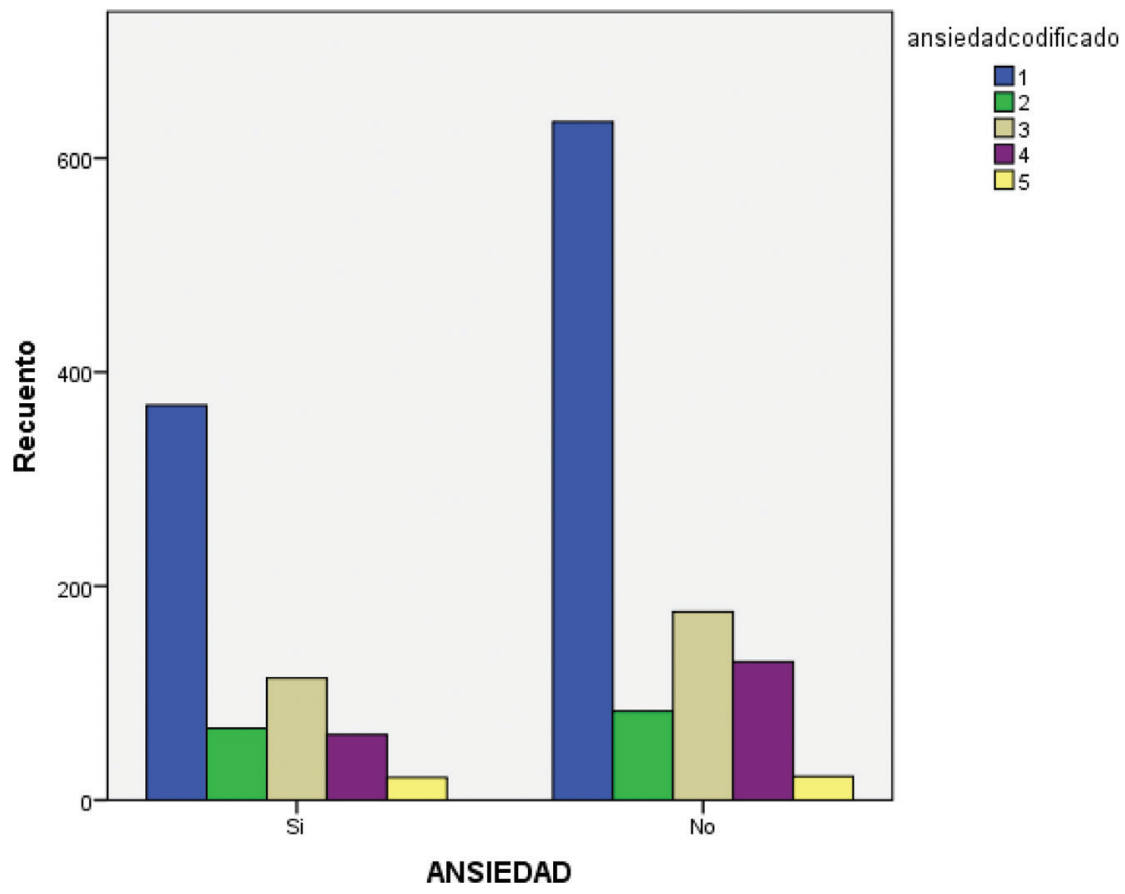

Figura 2. Asociación entre antecedentes de ansiedad y síntomas actuales.

salud mental, por lo que se debería contar con estrategias de información acerca de los síntomas más comunes en los padecimientos más frecuentes y de esta manera orientar a la población para estar más alerta y disponer de estrategias de autodetección, para que se acorte el tiempo entre la aparición de los síntomas y el diagnóstico de algún trastorno mental. 


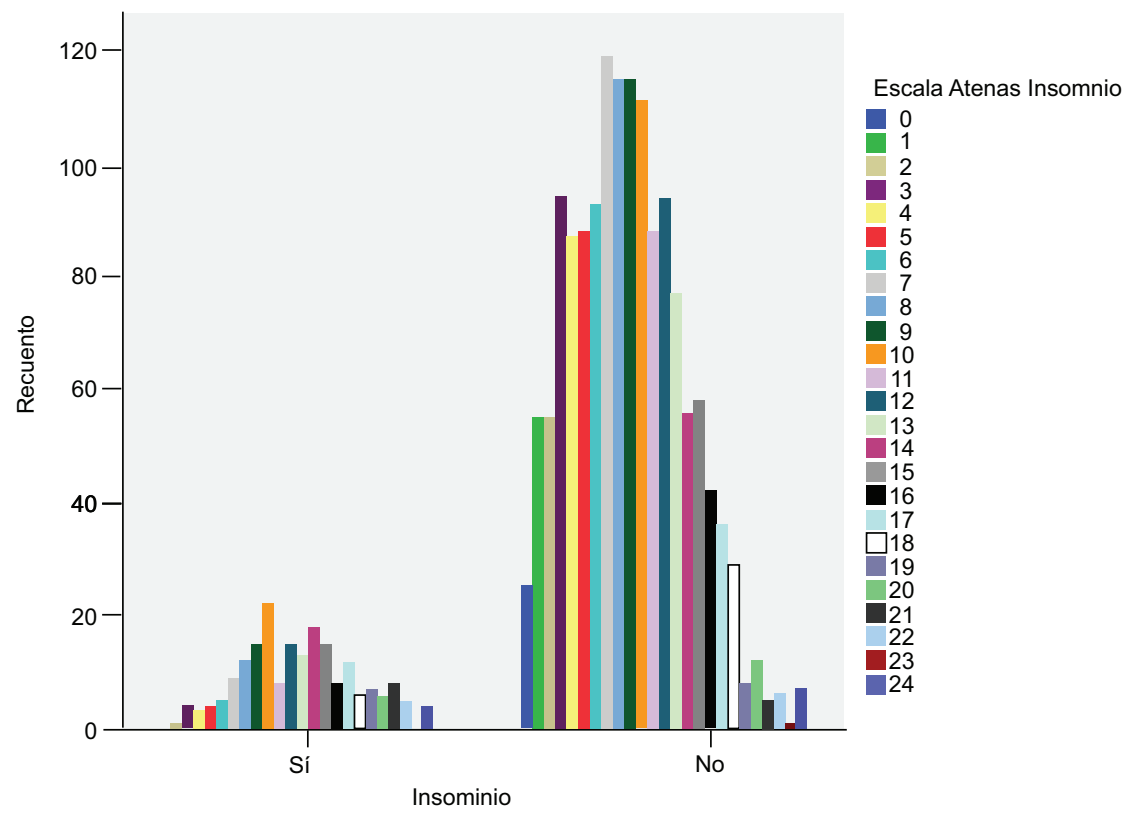

Figura 3. Asociación entre antecedentes de insomnio y síntomas actuales.

\section{Financiamiento}

Los autores no recibieron patrocinio para llevar a cabo este artículo.

\section{Conflicto de intereses}

Los autores declaran no tener conflicto de intereses alguno.

\section{Responsabilidades éticas}

Protección de personas y animales. Los autores declaran que para esta investigación no se realizaron experimentos en seres humanos ni en animales.

Confidencialidad de los datos. Los autores declaran que en este artículo no aparecen datos de pacientes.

Derecho a la privacidad y consentimiento informado. Los autores declaran que en este artículo no aparecen datos de pacientes.

\section{Referencias}

1. Pan American Health Organization [Internet]. EE. UU.: Mental health and psychosocial considerations during the COVID-19 outbreak; 2020.

2. World Health Organization [Internet]. Suiza: Q \& As on COVID-19 and related health topics; 2020.

3. Brooks SK, Webster RK, Smith LE, Woodland L, Wessely S, Greenburg N et al. The psychological impact of quarantine and how to reduce it: rapid review of the evidence. Lancet. 2020:395:912-920.

4. Rehman U, Shahnawaz MG, Khan NH, Kharshiing KD, Khursheed M, Gupta K, et al. Depression, anxiety and stress among indians in times of Covid-19. Community Ment Health J. 2020;1-7.
5. Taylor S, Landry CA, Paluszek MM, Fergus TA, McKay D, Asmundson GJG. Development and initial validation of the COVID stress scales. J. Anxiety Disord. 2020;72:102232.

6. Gao J, Zheng P, Jia Y, Chen H, Mao Y, Chen S, et al. Mental health problems and social media exposure during COVID-19 outbreak. PLoS One. 2020;15:e0231924.

7. Garfin DR, Silver RC, Holman EA. The novel coronavirus (COVID-2019) outbreak: amplification of public health consequences by media exposure. Health Psychol. 2020;39:355-357.

8. Pan American Health Organization [Internet]. EE. UU.: United Nation policy brief: COVID-19 and the need for action on mental health; 2020.

9. Bobo HPL, Chan CLW, Ng SM. Resilience of Hong Kong people in the COVID-19 pandemic: lessons learned from a survey at the peak of the pandemic in Spring 2020. Asia Pacific Journal of Social Work and Development. 2020;31:1-10.

10. Bonanno GA. Loss, trauma, and human resilience: Have we underestimated the human capacity to thrive after extremely aversive events? Am Psychol. 2004;59:20-28.

11. Bonanno GA, Galea S, Bucciarelli A, Vlahov D. Psychological resilience after disaster: New York City in the aftermath of the September $11^{\text {th }}$ terrorist attack. Psychol Sci. 2006;17:181-186.

12. Yuqing $Z$, Yuanyuan $A$, Xing $T$, Xiaohui L. Mental health and its influencing factors among self-isolating ordinary citizens during the beginning epidemic of COVID-19. J Loss Trauma. 2020;25:580-593.

13. Huang Y, Zhao N. Generalized anxiety disorder, depressive symptoms and sleep quality during COVID-19 outbreak in China: a web-based cross-sectional survey. Psychiatry Res. 2020;288:112954.

14. Phillipou A, Meyer D, Neill E, Tan EJ, Toh WL, van Rheenen TE, et al. Eating and exercise behaviors in eating disorders and the general population during the COVID 19 pandemic in Australia: Initial results from the COLLATE project. Int J Eat Disord. 2020;53:1158-1165.

15. Stanton R, To QG, Khalesi S, Williams SL, Alley SJ, Thwaite TL, et al. Depression, anxiety and stress during COVID-19: associations with changes in physical activity, sleep, tobacco and alcohol use in Australian adults. Int J Environ Res Public Health. 2020;17:4065.

16. Fitzpatrick KM, Harris C, Drawve G. How bad is it? Suicidality in the middle of the COVID-19 pandemic. Suicide Life Threat Behav. 2020;50.

17. Yao $\mathrm{H}$, Chen JH, Xu YF. Patients with mental health disorders in the COVID-19 epidemic. Lancet Psychiatry. 2020;7:e21.

18. Frank A, Hörmann S, Krombach J, Fatke B, Holzhuter F, Frank W, et al. Psychisch krank in Krisenzeiten: subjektive belastungen durch COVID-19. Psychiatry Prix. 2020;47:267-272

19. Hao F, Tan W, Jiang L, Zhang L, Zhoa X, Zou Y, et al. Do psychiatric patients experience more psychiatric symptoms during COVID-19 pandemic and lockdown? A case-control study with service and research implications for immunopsychiatry. Brain Behav Immun. 2020;87:100-106 
20. Palomar LJ, Gómez VN. Desarrollo de una escala de medición de la resiliencia con mexicanos (RESI-M). Interdisciplinaria. 2010;27:7-22.

21. Fahad A, Sandman MA. Evaluation resiliency patterns using the ER89: a case study form Kuwait. Social Behav Personality. 2000;28:505-514.

22. González-Arratia-López-Fuentes NI, Valdez-Medina JL. Resiliencia: diferencia por edad en hombres y mujeres mexicanos. Acta Invest Psicol. 2013:3:941-955.

23. Ustun G. Determining depression and related factors in a society affected by COVID-19 pandemic. Int J Soc Psychiatry. 2020;20764020938807.
24. Salari N, Hosseinian-Far A, Jalali R, Vaisi-Raygani A, Rasoulpoor S, Mohammadi M, et al. Prevalence of stress, anxiety, depression among the general population during the COVID-19 pandemic: a systematic review and meta-analysis. Global Health. 2020;16:57.

25. Voitsidis P, Gliatas I, Bairachtari V, Papadopoulou K, Papageorgiou G, Parlapani E, et al. Insomnia during the COVID-19 pandemic in a Greek population. Psychiatry Res. 2020;289:113076.

26. Kokou-Kpolou CK, Megalakaki O, Laimou D, Kousouri M. Insomnia during COVID-19 pandemic and lockdown: prevalence, severity, and associated risk factors in French population. Psychiatry Res. 2020;290:113128. 